\title{
Empagliflozin-one step closer to glycaemic control in patients with type II diabetes and CKD?
}

$\mathrm{T}$ he sodium-glucose cotransporter 2 (SGLT2) inhibitor, empagliflozinas an adjuvant to existing therapies -improves glycaemic control, and reduces body weight and blood pressure in patients with type II diabetes mellitus (T2DM) and stage 2 or stage 3 chronic kidney disease (CKD), according to the results of a phase III randomized controlled trial recently published in The Lancet Diabetes \& Endocrinology. The estimated glomerular filtration rates (eGFRs) and urinary albumin to creatinine ratios in these patients were also reduced with empagliflozin and, importantly, the researchers report that the drug was well-tolerated over a 52 week period.

Diabetes is a leading cause of CKD and effective control of blood glucose levels in diabetic patients is important to slow the decline in renal function and minimize the risk of complicationsT2DM and CKD are both risk factors for cardiovascular disease. SGLT2 is expressed exclusively in the proximal convoluted tubule and is responsible for the majority of glucose reabsorption in the nephron.

"SGLT2 inhibitors block the reabsorption of filtered glucose in the kidney proximal tubule, thereby increasing urinary glucose excretion and lowering blood glucose levels," explains Carol Pollock, Professor at the Kolling Institute of Medical Research in Sydney, Australia, who was not involved in the study. Her colleague Usha Panchapakesan continues, "given the mode of action of these inhibitors, two questions arise-are they effective antidiabetic agents in patients with renal impairment and can they offer renoprotection, beyond the improvement expected with glycaemic control?"

In the EMPA-REG RENAL trial, patients in 15 countries with T2DM and $\mathrm{CKD}$, in whom existing treatments were unsuccessful in controlling blood glucose, were randomly assigned to receive either empagliflozin or placebo in addition to

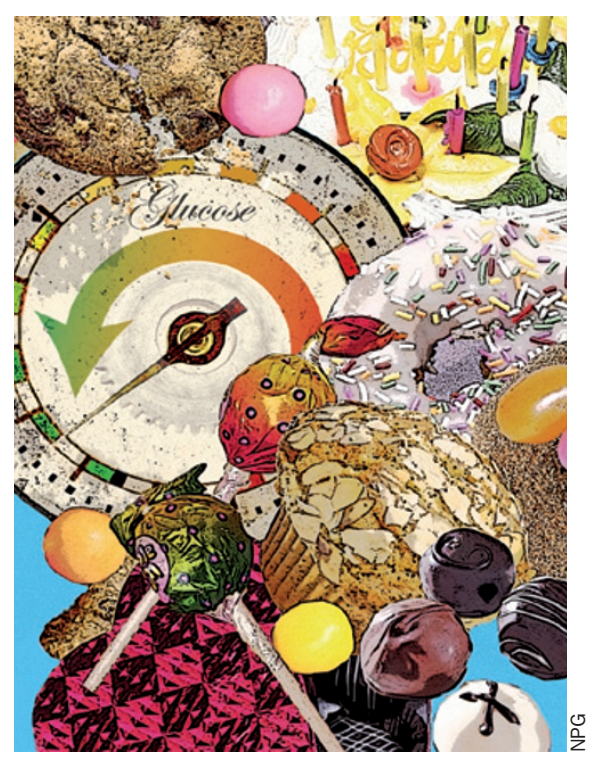

their existing antidiabetic treatments. Overall, 290 patients with stage $2 \mathrm{CKD}$ (eGFR $60-90 \mathrm{ml} / \mathrm{min} / 1.73 \mathrm{~m}^{2}$ ) received either placebo or empagliflozin (10 mg or $25 \mathrm{mg}$ ) and 374 patients with stage $3 \mathrm{CKD}$ (eGFR $30-60 \mathrm{ml} / \mathrm{min} / 1.73 \mathrm{~m}^{2}$ ) received either placebo or $25 \mathrm{mg}$ empagliflozin daily for 52 weeks.

At week 24, the adjusted mean difference in change from baseline haemoglobin A1c (HbA1c) level in patients with stage 2 CKD treated with $10 \mathrm{mg}$ empagliflozin versus placebo was $-0.52 \%$ (95\% CI -0.72 to $-0.32, P<0.0001$ ); for patients treated with $25 \mathrm{mg}$ empagflozin versus placebo this difference was $-0.68 \%$ (95\% CI -0.88 to $-0.49, P<0.0001)$. In patients with stage $3 \mathrm{CKD}$, the adjusted mean treatment difference in change from baseline $\mathrm{HbA} 1 \mathrm{c}$ level versus placebo at week 24 was $-0.42 \%$ (95\% CI -0.56 to $-0.28, P<0.0001$ ). Reductions in body weight, systolic blood pressure, and diastolic blood pressure were also reported with both doses of empagliflozin at week 24 and week 52 .

Small decreases in eGFR were reported in the groups treated with empagliflozin. However, eGFR returned to baseline levels within 3 weeks of treatment discontinuation, indicating no long-term detrimental effect of the drug on kidney function. In a linked commentary in the same journal, Piero Ruggenenti and Giuseppe Remuzzi state: "this finding also suggested that reversible eGFR and albuminuria reductions during treatment with this SGLT2 inhibitor could be explained by haemodynamic changes sustained by increased $\mathrm{NaCl}$ delivery to the macula densa induced by SGLT blockade."

Notably, adverse effects were comparable between patients treated with empagliflozin and placebo. Indeed, the trial investigators show that "empagliflozin, when added to existing medications in patients with stage 2 and stage $3 \mathrm{CKD}$, is safe and effective in lowering levels of HbA1c," comments Panchapakesan. However, as renal function declines, the efficacy in improving glycaemic control is reduced. "There remains a lack of evidence for treatment efficacy in patients with stage $4 \mathrm{CKD}$, when alternative oral hypoglycaemic agents are likely to have unacceptable adverse effects," says Pollock.

If future studies confirm nephroprotective and cardioprotective effects, without serious adverse effects, combination treatment that includes empagliflozin or other SGLT2 inhibitors might become the standard of care for patients with T2DM.

Bryony Jones

\footnotetext{
Original article Barnett, A. H. et al. Efficacy and safety of empagliflozin added to existing antidiabetes treatment in patients with type 2 diabetes and chronic kidney disease: a randomised, double-blind, placebo-controlled trial. Lancet Diabetes Endocrinol. doi:10.1016/ S2213-8587(13)70208-0

Further reading Ruggenenti, P. \& Remuzzi, G. Dreaming of normoglycaemia with fewer diet restrictions. Lancet Diabetes Endocrinol. doi:10.1016/S22138587(14)70002-6
} 
CORRECTION

Chronic kidney disease: Empagliflozin-one step closer to glycaemic control in patients with type II diabetes and CKD? Bryony Jones

Nat. Rev. Nephrol. advance online publication 18 February 2014; doi:10.1038/nrneph.2014.20

In the version of this article initially published online, Carol Pollock's name was incorrectly spelled. The error has been corrected for the print, HTML and PDF versions of the article. 